\title{
The transseptal approach for ablation of cardiac arrhythmias: experience of 104 procedures
}

\author{
N J Linker, A P Fitzpatrick
}

\begin{abstract}
Objective-To report retrospectively on the training and subsequent experience of two operators in transseptal ablation of arrhythmias arising in the left atrium and left atrioventricular annulus, to show whether, with adequate training and careful attention to detail, this is a safe and effective technique.

Setting-Electrophysiological studies and transseptal procedures were performed in the electrophysiology laboratories of the Moffatt Hospital, University of California at San Francisco (39) and Manchester Royal Infirmary (65) from January 1993 to June 1997. Close supervision by a fully trained operator was provided for at least the first 20 procedures performed by each operator.

Patients-94 consecutive patients underwent electrophysiological study and ablation for Wolff-Parkinson-White syndrome with left sided accessory connections (81 patients) or ectopic atrial tachycardia (13 patients); 104 transseptal procedures were done; eight patients required multiple procedures.
\end{abstract}

Results-92 patients (98\%) were initially successfully ablated. Five of 81 with accessory pathways $(6 \%)$ and three of 13 with atrial tachycardia (23\%) required further procedures. One patient with WolffParkinson-White syndrome could not be ablated at a second procedure. Long term success rate for accessory pathway ablation was therefore $99 \%$. Procedures were abandoned in three patients because of minor complications. All were subsequently ablated successfully by a transseptal approach on another day.

Conclusions-The transseptal approach is safe and effective for ablation of left sided arrhythmias. The technique has similar success rates to the retrograde transaortic approach but without the risk of inadvertent damage to the coronary arteries or aortic valve.

(Heart 1998;79:379-382)

Keywords: arrhythmias; accessory pathways; transseptal ablation

Catheter ablation of arrhythmias has been attempted in various ways since the first description of the technique in $1983 .{ }^{1}$ There has been considerable experience and success in the ablation of left sided accessory pathways using the retrograde transaortic approach. ${ }^{2}{ }^{3}$ However, the 1992 NASPE survey ${ }^{4}$ found a significant complication rate of $2.1 \%$ and a procedure related death rate of $0.2 \%$, caused mainly by the need for retrograde left heart arterial catheterisation. It was also noted in that survey that data on complication rates were incomplete.

Certain centres have reported high success rates and few complications using the transseptal approach for left sided accessory pathways. ${ }^{56}$ In this paper we describe the early training and experience of two operators in ablating accessory pathways and left atrial ectopic tachycardias with this technique.

\section{Methods}

PATIENTS

Ninety four patients (39 male, mean age 34 years, range 5 to 71 ) have undergone 104 radiofrequency ablation procedures using a transseptal approach. Before diagnostic electrophysiological testing, patients presented with either manifest Wolff-Parkinson-White syndrome (57), or paroxysmal or incessant supraventricular tachycardia (PSVT) (37). Patients with Wolff-Parkinson-White syndrome had manifest left anterolateral, left posterolateral, or left posteroseptal accessory pathways, as previously defined. ${ }^{7}$ Of patients with PSVT, 24 had orthodromic tachycardia using a concealed accessory pathway, and 13 had an ectopic atrial tachycardia. Of the 94 patients, 37 were studied in San Francisco and 57 in Manchester.

\section{PROCEDURES}

Diagnostic electrophysiological study

For the diagnostic portion of a combined electrophysiological study and subsequent ablation, patients were fasted for at least four hours. A decapolar electrode was positioned in the coronary sinus with the position confirmed by contrast venography. Quadripolar electrodes were positioned to record the His potential and placed in the right atrial appendage and right ventricular apex. The diagnostic study techniques are described in detail elsewhere. ${ }^{8}$ Diagnostic electrophysiological testing confirmed a left sided accessory pathway or ectopic atrial tachycardia focus in all cases.

\section{Fluoroscopic landmarks}

The coronary sinus and His bundle electrodes provide important anatomical markers to locate the coronary sinus ostium and the right side of the membranous septum. It is customary to place a pigtail catheter in the aortic root for transseptal punctures for mitral valvoplasty, and this was done for the first 40 procedures in this series. However, we learned during this experience that this was not necessary, since 



Figure 1 (A) Right anterior oblique $45^{\circ}$ projection showing the ablation catheter $(A b l)$ lying on the mitral valve annulus through a transseptal Swartz SL2 sheath (TSP sheath). Some staining of the interatrial septum is seen owing to previous contrast injections.

(B) Left anterior oblique $40^{\circ}$ projection showing the ablation catheter (Abl) lying on the mitral valve annulus through a transseptal Swartz SL2 sheath (TSP sheath). Some staining of the interatrial septum is seen due to previous contrast injections.

the aortic valve lies immediately above the membranous septum on the left side, and the His bundle runs over this part of the septum. The His catheter therefore lies just a few millimetres from the aortic valve, providing a safe fluoroscopic marker. Furthermore, when the transseptal assembly is drawn down the interatrial septum towards the fossa ovalis (see below) there is an abrupt septal movement of the tip as it "pops" into the fossa. However, if the plane of the transseptal assembly curve is incorrect, and the tip too low, this same appearance can occur as the tip falls into the ostium of the coronary sinus. Therefore positioning of both the His and coronary sinus catheters is crucial to safe performance of the puncture.

\section{Transseptal technique}

Throughout the diagnostic electrophysiological study and transseptal procedure the patients were kept well hydrated with a continuous infusion of normal saline, receiving at least $500 \mathrm{ml}$ before the transseptal procedure. The transseptal procedures were performed using a standard Brockenborough needle and either a Mullins sheath (Bard Inc, Billerica, Massachusetts, USA) or a Swartz sheath and dilator (Daig Corporation, Minnetonka, Minnesota, USA). ${ }^{5}$ The needle was connected to a standard catheterisation manifold by a short length of flexible tubing, so that contrast could be injected and pressure could be displayed and recorded. Biplane fluoroscopic projections used were (right anterior oblique $45^{\circ}$ and left anterior oblique $40^{\circ}$ ) in all cases, providing en face and longitudinal views of the interatrial septum respectively. The transseptal sheath/ dilator was placed in the superior vena cava and then the dilator/sheath/needle combination was withdrawn inferiorly in the left anterior oblique projection until the tip of the dilator "popped" into the fossa ovalis. Positioning was always checked in the right anterior oblique projection to ensure that the tip of the needle/sheath assembly was pointing directly away from the plane of vision and slightly anterior. A test injection of contrast was delivered through the dilator in the left anterior oblique projection to check for "tenting" of the fossa at the intended puncture site. This was typically just below the level of the His recording catheter in the left anterior oblique $40^{\circ}$ projection, and well behind the His catheter electrodes in the right anterior oblique $45^{\circ}$. The needle was then advanced and a second test injection was made following needle advancement to check that the needle had entered the left atrium; left atrial pressure was then recorded. The sheath/dilator were advanced over the needle into the left atrium. Once the dilator was well into the left atrium, the sheath was advanced over the dilator, but the needle remained just inside the dilator to give support for advancement of the long sheath. Once the needle is withdrawn, the tip of the dilator tends to flex. This makes it hazardous to redeploy the needle, since this may result in penetration of the wall of the dilator.

Patients then received 5000 units of heparin, with a further 2500 units being given if left sided deployment lasted more than one hour. If deployment exceeded 90 minutes, activated partial thromboplastin time was estimated. Ablations were performed using $4 \mathrm{~mm}$ tipped "Steerocath" or "Blazer-T" thermistor tipped electrodes (EP Technologies, Sunnyvale, California, USA). The ablation catheter was passed through the sheath into the left atrium and positioned on the mitral annulus (fig 1A and B). Care was always taken to flush the sheath during catheter advancement to avoid entrainment of air. Suitable ablation sites were obtained using conventional criteria. ${ }^{6}$ Patients remained in the laboratory with transseptal sheath in place for 30 minutes after the proce- 
Table 1 Patient characteristics and results

\begin{tabular}{llll}
\hline Condition & Number & $\begin{array}{l}\text { Immediate } \\
\text { success }\end{array}$ & $\begin{array}{l}\text { Long term } \\
\text { success (\%)* }\end{array}$ \\
\hline WPW & 81 & 81 & $80(98)$ \\
EAT & 13 & 12 & $12(92)$ \\
\hline
\end{tabular}

${ }^{\star}$ More than three months' follow up.

WPW, Wolff-Parkinson-White syndrome with concealed or manifest pre-excitation; EAT, ectopic atrial tachycardia arising from left atrium.

dure (particularly after accessory pathway ablation) for confirmation of success. After successful ablation, transseptal sheaths were withdrawn across the interatrial septum with the ablation catheter tip protruding slightly. This technique was employed to avoid inadvertent displacement of adherent coagulum from the catheter at the sheath tip.

\section{Results}

Of the 81 patients with left sided accessory pathways, initial successful ablation of the pathway was seen in all patients at the end of the procedure (table 1). Recurrence was observed in five patients $(6 \%)$, four young (mean (SD) age, 12 (3) years), and one woman of 56 years, all initially ablated using a Mullins sheath. In all but the older patient ablation was successful on a second or third occasion using a transseptal approach and an appropriately shaped Swartz sheath. Successful ablation of left atrial tachycardia was accomplished in all patients. Four required multiple procedures. One required three attempts, each through a transseptal puncture. In this patient, the automatic tachycardia was found to be arising from a discrete aneurysm of the left atrium. One elderly woman had recurrence of a left atrial tachycardia four months after successful ablation and was later treated by atrioventricular nodal ablation and pacing.

The mean (SD) total fluoroscopy time was 31 (20) minutes (25 (13) minutes for patients with accessory pathways), the mean total procedure time (time from arrival in the catheter laboratory to return to the ward) was 117 (33) minutes (112 (26) minutes for patients with accessory pathways), and the mean left atrial "dwell" time was 47 (15) minutes. In one patient (during the training of $\mathrm{APF}$ ), staining of the posterior atrial septum with contrast injection was observed and caused chest pain, so the procedure was abandoned. The patient was observed overnight and then restudied and successfully ablated by a transseptal route the following day. In two patients (one treated by APF, the other by NJL, both after the initial training period, and both at the Manchester Royal Infirmary), the sheath was deployed but it was not possible to aspirate or record pressure through it. Fluoroscopic location appeared satisfactory. In both cases the sheath was withdrawn and the procedure abandoned. There were no adverse sequelae, and both patients underwent successful ablation by a transseptal route some days later.

\section{Discussion}

Radiofrequency catheter ablation of left sided accessory pathways has been most commonly performed using a retrograde transaortic approach. ${ }^{21011} \mathrm{~A}$ few centres, particularly in the USA, have reported experience using the transseptal approach. ${ }^{569}$ Similar success and complication rates have been observed between the two techniques in the reported series. In this study, there were no important complications with the transseptal approach, but success rates are comparable with others.

With this technique there are several likely reasons for the lack of complications. Firstly, rigorous training was provided. Both operators (who are experienced catheterisers) were closely supervised for their first 20 procedures by a senior operator scrubbed for the procedure. Attention to detail was emphasised, and the use of biplane fluoroscopy to ensure accurate needle/sheath assembly placement, accurate contrast injection, and minimisation of the time spent in the left side of the heart are all important considerations.

There are various advantages of the transseptal technique over the retrograde approach. The rove/ablation catheter is much more easily positioned in the left atrium owing to the absence of the subvalvar apparatus. The mitral annulus is quite well formed on the atrial side, providing a stable target for catheter positioning. Other workers have noted a shorter duration of fluoroscopy and a reduction in total procedure time with the transseptal technique. ${ }^{6}$ The sheath facilitates manipulation of the ablation catheter along the mitral annulus and provides added benefits even if a patent foramen ovale is present. The use of sheaths with a variety of single and biplanar curves specifically designed for the transseptal approach greatly facilitates the firm siting of the ablation electrode on high, intermediate, low, and paraseptal aspects of the mitral annulus. We attribute postprocedure recurrence of pathway conduction in five young patients with Wolff-Parkinson-White syndrome to the use of Mullins sheaths, and later success to the use of Swartz sheaths, which provide better catheter stability and tissue contact. The retrograde approach usually requires catheter placement underneath the mitral valve leaflets. In contrast, in the transseptal approach energy is delivered on the atrial side. This is also helpful in that the physical irritation of ventricular myocardium by the ablation catheter, with resultant troublesome ventricular ectopy, is avoided.

No important sequelae of the transseptal technique were observed in this series, although three procedures had to be abandoned. Using the retrograde transaortic approach, problems have been reported in relation to arterial access, problems related to catheter manipulation within the coronary ostia, and catheter manipulation across the aortic valve. ${ }^{61012}$ Griffith et al reported their experience in 25 patients who underwent retrograde catheter ablation of left sided accessory pathways and noted four cases of aortic regurgitation (16\%), although three were only detectable using Doppler echocardiography, and occurred early in the series. ${ }^{13}$ Complications related to the aortic valve and coronary 
ostia should be avoided with the transseptal approach. A theoretical concern of the potential arrhythmogenic effect of radiofrequency energy on ventricular myocardium ${ }^{9}$ is also avoided by approaching the mitral annulus from the atrial side.

There are potential problems with the transseptal technique, including inadvertent puncture of the aorta and pulmonary arteries; puncture into the pericardial space may also occur. Adequately supervised training in this technique is mandatory, with careful attention to anatomical location using catheters/ electrodes as markers, as described above. With the decline in use of the transseptal technique in diagnostic cardiology for assessment of valvar heart disease, there is likely to be a decreasing pool of experienced operators. We believe the key to success and safety is proper training, good supervision, and attention to detail. With these provisos, the use of the transseptal technique as an approach to the left side of the heart for the management of left sided accessory atrioventricular connections and other left sided arrhythmias is a safe and effective.

1 Weber H, Schmitz L. Catheter technique for closed chest ablation of an accessory atrioventricular pathway. $N$ Engl f Med 1983;308:653-4.
2 Jackman WM, Wang X, Friday KJ, et al. Catheter ablation of accessory atrioventricular pathways (Wolff-ParkinsonWhite syndrome) by radiofrequency current. $\mathrm{N} \mathrm{Engl} \mathrm{f} \mathrm{Med}$ 1991;324:1605-11.

3 Schluter M, Geiger M, Siebels J, et al. Catheter ablation using radiofrequency current to cure symptomatic patients with tachyarrhythmias related to an accessory atrioventricular pathway. Circulation 1991;84:1644-61.

4 Scheinman MM. Patterns of catheter ablation practice in the United States: results of the 1992 NASPE survey. PACE 1994;17:873-5.

5 Swartz JF, Tracy CM, Fletcher RD. Radiofrequency endocardial catheter ablation of accessory atrioventricular

Dash MD, Van Hare G, Sche of the retrograde and trans-septal methods for ablation of of the retrograde and trans-septal methods for ablation of
left free wall accessory pathways. $7 \mathrm{Am}$ Coll Cardiol 1993;22:542-9.

7 Fitzpatrick AP, Gonzales RG, Lesh MD, et al. New algorithm for the localization if accessory atrioventricular connections using a baseline electrocardiogram. $\mathcal{F} \mathrm{Am}$ Coll Cardiol 1994;23:107-16.

8 Josephson ME. Clinical cardiac electrophysiology, 2nd ed. Philadelphia: Lea and Febiger, 1992:22-70.

9 Manolis AS, Wang PJ, Estes NA. Radiofrequency ablation of left-sided accessory pathways: transaortic versus transseptal approach. Am Heart f 1994;128:896-902.

10 Calkins H, Langberg J, Sousa J, et al. Radiofrequency catheter ablation of accessory atrioventricular connections in 250 patients: abbreviated therapeutic approach to WolffParkinson-White syndrome. Circulation 1992;85: 1337-46.

11 Kuck K-H, Schluter M, Giger M, et al. Radiofrequency current catheter ablation of accessory atrioventricular pathways. Lancet 1991;337:1557-61.

12 Thakur RK, Klein GJ, Yee R, et al. Complications of radiofrequency catheter ablation: a review. Can $\mathcal{f}$ Cardiol 1994;10:835-9.

13 Griffith MJ, Kullar K, Jordan PJ. The learning curve for radiofrequency ablation is more than 160 procedures [abstract]. Br Heart f 1996;75:P44. 